\title{
Open-Label Study to Assess the Efficacy of Ipragliflozin for Reducing Insulin Dose in Patients with Type 2 Diabetes Mellitus Receiving Insulin Therapy
}

\author{
Hisamitsu Ishihara ${ }^{1}$ (D) Susumu Yamaguchi ${ }^{2} \cdot$ Toshifumi Sugitani $^{2} \cdot$ Yoshinori Kosakai $^{2}$
}

Published online: 24 September 2019

(c) The Author(s) 2019

\begin{abstract}
Background and Objective To avoid insulin-induced hypoglycemia and weight gain, the minimum dose of insulin should be used. In this study, therefore, we examined insulin dose reduction by ipragliflozin add-on therapy in Japanese patients with type 2 diabetes mellitus treated with long-acting basal insulin.

Methods In this multicenter, open-label study, patients received one ipragliflozin 50-mg tablet once daily in combination with basal insulin for 24 weeks. The primary efficacy endpoint was the change and percent change in insulin dose from baseline to Week 24. Secondary efficacy endpoints included changes in glycated hemoglobin (HbA1c), fasting plasma glucose (FPG), glycoalbumin, cholesterol, leptin, adiponectin, C-peptide, glucagon, body weight, and blood pressure, and number of patients achieving withdrawal of insulin at the end of treatment (EOT). Treatment-emergent adverse events (TEAEs) were evaluated for safety.

Results In total, 114 patients were screened, 103 were registered, and 97 completed the study. The mean age was 59 years and $72.8 \%$ of patients were male. The mean change in insulin dose from baseline at Week 24 was $-6.6 \pm 4.4$ units/day $(p<0.001)$; the mean percent change was $-29.87 \%$. HbA1c, FPG, glycoalbumin, glucagon levels, body weight, and blood pressure significantly decreased from baseline to EOT $(p<0.05)$. Cholesterol, leptin, and adiponectin were unaffected. One patient was able to stop insulin treatment at Week 16. The incidence of TEAEs was $60.2 \%$. Hypoglycemia (10.7\%) and pollakiuria (13.6\%) were the most common drug-related TEAEs. Conclusions Once-daily 50-mg ipragliflozin enabled a 30\% dose reduction of insulin by Week 24 compared with baseline. No major safety concerns were raised.
\end{abstract}

Clinical Trial Registration ClinicalTrials.gov (NCT02847091)

\section{Introduction}

Treatment of type 2 diabetes mellitus (T2DM) aims to achieve good glycemic control with glycated hemoglobin (HbA1c) serving as an indicator (or barometer) while avoiding hypoglycemia [1]. Weight control and normalizing blood pressure and blood lipid parameters are also important to

Electronic supplementary material The online version of this article (https://doi.org/10.1007/s40261-019-00851-z) contains supplementary material, which is available to authorized users.

Hisamitsu Ishihara

ishihara.hisamitsu@nihon-u.ac.jp

1 Division of Diabetes and Metabolic Diseases, Nihon University School of Medicine, 30-1 Oyaguchikami-cho, Itabashi-ku, Tokyo 173-8610, Japan

2 Astellas Pharma Inc., 2-5-1, Nihonbashi-honcho, Chuo-ku, Tokyo 103-8411, Japan prevent cardiovascular and renal complications. In Japan, approximately $30 \%$ of T2DM patients are treated with insulin [2], which is the oldest and most effective therapy for lowering blood glucose levels. However, insulin use has its own risks, such as insulin-induced hypoglycemia and weight gain [3,4], which may lead to poor adherence to therapy $[5,6]$. Thus, it is ideal to use a minimum necessary dose of insulin to mitigate unfavorable side effects. In contrast, a relatively new class of antihyperglycemic drugs, the sodiumglucose cotransporter-2 (SGLT2) inhibitors, have demonstrated efficacy in managing T2DM without inducing hypoglycemia or weight gain. In addition, large-scale randomized controlled studies have demonstrated the cardiovascular and renal benefits associated with this drug class [7, 8], moving these drugs to the center stage of oral diabetes agents.

Ipragliflozin, an SGLT2 inhibitor approved for the treatment of T2DM in Japan and Korea, has demonstrated efficacy in managing T2DM with a favorable safety profile, both as monotherapy and in combination with other oral 


\section{Key Points}

This open-label study investigated the extent to which 50 -mg, once-daily ipragliflozin, a sodium-glucose cotransporter- 2 inhibitor, can reduce insulin requirements in type 2 diabetes mellitus patients who have progressed to basal insulin therapy.

After 24 weeks of treatment, ipragliflozin elicited a mean (standard deviation) change in basal insulin dose of $-6.6 \pm 4.4$ units/day, reflecting a mean percent change of $-29.87 \%$, and one patient was able to stop insulin therapy entirely by Week 16 .

Once-daily ipragliflozin safely and effectively reduced the dose of insulin necessary to maintain acceptable glycemic control.

agents, insulin, or glucagon-like peptide-1 (GLP-1) receptor agonists $[9,10]$. Ipragliflozin has also been shown to elicit favorable effects on cardiovascular risk factors such as body weight, lipid parameters, uric acid, and blood pressure [11, 12]. SGLT2 inhibitors, when administered concomitant to insulin therapy, may have the potential to reduce the insulin dose necessary to achieve adequate glycemic control, leading to mitigation of hypoglycemia and insulin-related weight gain $[9,13,14]$. Indeed, previous retrospective studies in Canada [15] and India [16] have demonstrated that SGLT2 inhibitor add-on therapy to insulin brought about substantial reductions in insulin dose under real-world conditions. However, there exist no prospective studies that examine the magnitude of insulin-sparing effects of SGLT2 inhibitors.

Therefore, in the present study, our objective was to determine the extent to which ipragliflozin add-on therapy can reduce the insulin dose in Japanese T2DM patients treated with basal insulin. Patients underwent a prespecified insulin dose reduction of $20-40 \%$ starting from Day 1 (i.e. the first day of ipragliflozin administration), and we assessed the effect of once-daily, 50-mg ipragliflozin on overall change in insulin dose while maintaining glycemic control.

\section{Methods}

\subsection{Study Design and Treatments}

This multicenter, prospective, single-arm, open-label study was conducted in 15 centers in Japan (Online Resource 1) between 29 July 2016 and 9 November 2017. Patients with T2DM who had been receiving insulin therapy (insulin monotherapy with a long-acting formulation) for at least
12 weeks before Visit 1 (Week 0; baseline) were enrolled. In general, changes in the dose or type of insulin therapy were not allowed for 12 weeks before Visit 1 . The dose of insulin therapy could be changed $\pm 10 \%$ based on the daily dose 12 weeks before Visit 1 if clinically necessary, but the active ingredient had to remain unchanged. The treatment period lasted 24 weeks (Visits 1-8). During this period, one ipragliflozin 50-mg was administered once daily in combination with insulin therapy. Study visits were scheduled at Weeks $0,2,4,8,12,16,20$, and end of treatment.

To prevent hypoglycemia during the initial phase of combined treatment, the insulin dose was reduced by $20-40 \%$ at the start of ipragliflozin treatment (Visit 1). The baseline insulin dose was defined as the dose prior to this initial dose reduction. During the study, the daily insulin dose could be decreased if any hypoglycemic symptoms occurred, or if blood glucose levels dropped below $70 \mathrm{mg} /$ $\mathrm{dL}(3.89 \mathrm{mmol} / \mathrm{L})$ according to the self-monitored blood glucose level in the morning and if the treating physicians considered the patient to be at high risk of hypoglycemia from $\mathrm{HbA} 1 \mathrm{c}$ and blood glucose levels. Increases in insulin dose were required if blood glucose levels exceeded $180 \mathrm{mg} /$ $\mathrm{dL}(10 \mathrm{mmol} / \mathrm{L})$ over two consecutive measurements or allowed when the treating physician deemed dose increases necessary. Patients were asked to perform self-monitoring of blood glucose and record the result each morning in a daily compliance diary. Patients were also advised by the treating physician to exercise regularly and manage their diets without changing conditions.

Criteria for study withdrawal included major protocol deviation, severe hypoglycemia, serum creatinine exceeding 1.5 times the baseline value and exceeding $2.0 \mathrm{mg} / \mathrm{dL}$ ( $176.80 \mu \mathrm{mol} / \mathrm{L}$ ), estimated glomerular filtration rate (eGFR) based on the Japanese GFR estimation equation (the age at Visit 1 was used for calculation) falling below $20 \mathrm{~mL} /$ $\mathrm{min} / 1.73 \mathrm{~m}^{2}$, HbAlc level exceeding $11.5 \%$ (107 mmol/mol) at one visit or $10.5 \%(96 \mathrm{mmol} / \mathrm{mol})$ at two consecutive visits, or a single fasting plasma glucose (FPG) measurement exceeding $350 \mathrm{mg} / \mathrm{dL}(19.43 \mathrm{mmol} / \mathrm{L})$. Compliance with the assigned treatment was assessed by the investigator based on review of the compliance diary and patient interview and recorded in the medical records.

This study was conducted in accordance with the ethical principles of the Declaration of Helsinki, Good Clinical Practice, Good Post-marketing Surveillance Practice, and the International Council for Harmonization of Technical Requirements for Pharmaceuticals for Human Use Guidelines. All patients provided informed consent at Visit 1 before any study-related screening procedures. Institutional review boards at each site approved the study. 


\subsection{Patients}

Eligible patients were outpatients aged $\geq 20$ years and $<75$ years at the time of informed consent and who had T2DM that had been treated with $\geq 10$ units/day of long-acting insulin for $\geq 12$ weeks before Visit 1. Patients could have been prescribed one or two concomitant oral antihyperglycemic agents (dipetidyl peptidase- 4 inhibitors, biguanides, thiazolidinediones or $\alpha$-glucosidase inhibitors, but not sulfonylureas or glinides). Use of GLP-1 receptor agonists and insulins other than the long-acting formula were not allowed. Changing the type and dosage of oral antihyperglycemic agents during this study was prohibited. Further, antihypertensive drugs and lipid-lowering drugs for the treatment of comorbidities could be used concomitantly without any changes in the dosage. Patients also had to have a baseline HbA1c level between 6.5 and $<8.0 \%$ (47 mmol/ $\mathrm{mol}$ and $<63 \mathrm{mmol} / \mathrm{mol}$ ) based on in-hospital measurement and body mass index $(\mathrm{BMI})>23.0 \mathrm{~kg} / \mathrm{m}^{2}$. Patients of childbearing potential had to take measures not to get pregnant during the study; women had to agree not to breastfeed during the study.

Patients were excluded if they had type 1 diabetes mellitus, proliferative retinopathy, diabetic ketoacidosis, uncontrolled blood pressure (systolic blood pressure $>180 \mathrm{mmHg}$ or diastolic blood pressure $>110 \mathrm{mmHg}$, measured in the sitting position after a 5-min rest), severe hepatic dysfunction, or a history of heart or vascular disease, medically significant renal disease, or recurrent or currently symptomatic urinary tract infection. Patients were also excluded if they required continuous use of corticosteroids, immunosuppressants, or similar drugs.

\subsection{Efficacy}

The primary efficacy endpoint was the change and percent change in insulin dose from baseline to Week 24. Insulin dose was assessed at Weeks $0,2,4,8,12,16$, and 20, and the end of treatment. The secondary efficacy endpoints were the following: change and percent change in the insulin dose from baseline at each assessment time point (Weeks 0, 2, 4, $8,12,16$, and 20 , and the end of treatment), change in each outcome measure (HbA1c, FPG, glycoalbumin, cholesterol, leptin, adiponectin, C-peptide, glucagon, body weight, waist circumference, and blood pressure) from baseline at each assessment time point, changes in the Diabetes Treatment Satisfaction Questionnaire (DTSQ) from baseline at Week 24 and the end of treatment, and number and percentage of patients achieving withdrawal of insulin therapy at each assessment time point. All laboratory tests were conducted at a central laboratory (SRL Inc., Tokyo, Japan). Leptin was measured using radioimmunoassay, adiponectin using latex immune-turbidimetry, and glucagon using an enzyme-linked immunosorbent assay. The DTSQ assessment included items $1,4,5,6,7$, and 8, which comprise the Treatment Satisfaction subset of the tool [17]; items 2 and 3 of the DTSQ were excluded because they did not align with the study outcomes. An additional analysis was performed to assess the changes in insulin dose and $\mathrm{HbA} 1 \mathrm{c}$ from baseline to Week 24 as stratified by three baseline insulin dose categories $(<15,15$ to $<30$, and $\geq 30$ units/day).

\subsection{Safety}

The number of treatment-emergent adverse events (TEAEs), as well as the number and percentage of patients with TEAEs were summarized. AEs were coded by the Medical Dictionary for Regulatory Activities, Japanese version (MedDRA/J, v19.0). Hypoglycemia was defined as a fasting blood glucose level of $<60 \mathrm{mg} / \mathrm{dL}$. However, if patients reported any symptoms of hypoglycemia even at a fasting blood glucose of $\geq 60 \mathrm{mg} / \mathrm{dL}$, the event was defined as hypoglycemia. The treating physician judged whether an AE had occurred.

\subsection{Statistical Methods}

Previous studies that assessed the efficacy of concomitant SGLT2 inhibitor use in reducing the insulin dose showed that the standard deviation (SD) of the change in the insulin dose was approximately $15-25 \%$ at higher baseline doses [18-20]. As the SD of the change tended to increase in proportion to the baseline insulin dose, the SD in the target patients in this study was considered to be below 15 . Therefore, the planned sample size was 100 patients, with an estimated standard error of approximately $\leq 1.5$, and this corresponded to approximately $10 \%$ of the mean baseline dose.

The full analysis set was used for the efficacy analyses and comprised patients who received $\geq 1$ dose of ipragliflozin and who had a baseline insulin dose measurement, as well as $\geq 1$ post-baseline insulin measurement. Patients in the per-protocol analysis set included those in the full analysis set who had $>80 \%$ compliance rate for ipragliflozin, no prohibited or conditionally allowed concomitant medication use, and no major protocol deviations. Patients in the safety analysis set received $\geq 1$ dose of ipragliflozin.

For the primary endpoint analysis, descriptive statistics were calculated for the insulin dose at Week 24 and the change and percent change in insulin dose from baseline at Week 24. Means and SDs of measured values and changes from baseline were plotted. In the secondary endpoint analyses, descriptive statistics were calculated for measured values and changes and percent changes from baseline at each assessment time point. For both primary and secondary endpoints, $p$ values were calculated for the change in insulin dose from baseline, using Wilcoxon's signed rank 
test. All $p$ values less than 0.05 were considered statistically significant. The number and percentage of patients achieving withdrawal of insulin therapy were summarized.

The number of TEAEs as well as number and percentage of patients with TEAEs, classified by system organ class and preferred term, were summarized in the safety analysis set. The statistical analysis was performed using SAS ${ }^{\circledR}$ Studio 3.5 (Cary, NC, USA).

\section{Results}

\subsection{Patients}

A total of 100 patients were planned for inclusion: 114 were screened; 103 were registered in the study; and 97 completed the study (Online Resource 2). All patients who were registered in the study took the study drug; thus, the full analysis and safety analysis sets consisted of 103 patients each, whereas 100 patients comprised the per-protocol set.

Patient characteristics are shown in Online Resource 3. Most patients were male $(72.8 \%)$. The mean age was 59 years, with approximately $30 \%$ of patients older than 65 years. The mean body weight was $73.92 \mathrm{~kg}$, and the mean BMI was $26.76 \mathrm{~kg} / \mathrm{m}^{2}$. The patients had a mean duration of diabetes mellitus of approximately 163 months, with a mean $\mathrm{HbA} 1 \mathrm{c}$ level of $7.62 \%(59.7 \mathrm{mmol} / \mathrm{mol})$ and a mean FPG level of $129.2 \mathrm{mg} / \mathrm{dL}(7.17 \mathrm{mmol} / \mathrm{L})$. The mean $\pm \mathrm{SD}$ duration of ipragliflozin therapy was $164 \pm 26$ days. All patients had $>80 \%$ treatment compliance and the mean \pm SD treatment compliance was $99.68 \% \pm 0.72 \%$. The patients were prescribed insulin glargine U-100 (59.2\%), insulin glargine U-300 (7.8\%), insulin degludec (30.1\%), or insulin detemir $(2.9 \%)$.

\subsection{Efficacy}

\subsubsection{Primary Endpoint}

The mean \pm SD baseline insulin dose in the full analysis set was $23.0 \pm 12.6$ units/day and was reduced by $6.6 \pm 4.4$ units/ day at Week 24 of ipragliflozin treatment (Fig. 1a), which corresponded to $29.87 \%$ of the baseline value (Fig. 1b). The $p$ value of the Wilcoxon's signed rank test for the change in the insulin dose was $<0.001$. The time course data show a sharp decrease between baseline and Visit 2; this included the initial $20-40 \%$ dose reduction that was applied at the beginning of the study (i.e. approximately 6 units/day), which was maintained for 24 weeks. A similar result was seen with the per-protocol analysis set.

Figure 1c illustrates the percent changes in insulin dose stratified by three baseline insulin dose categories $(<15$, 15 to $<30$, and $\geq 30$ units/day). Reductions in insulin dose were observed in all three baseline insulin dose categories (all $p<0.001$ ), and the percent reductions were similar across all three categories. We also analyzed the percent reduction in insulin dose stratified across three baseline eGFR categories. We observed $-25.73 \% \pm 6.86 \%(n=14)$, $-31.37 \% \pm 20.20 \%(n=53)$, and $-29.14 \% \pm 13.72 \%$ $(n=29)$ changes in patients categorized by baseline eGFR levels of 30 to $<60,60$ to $<90$, and $\geq 90 \mathrm{~mL} / \mathrm{min} / 1.73 \mathrm{~m}^{2}$, respectively. Thus, there were similar significant reductions in insulin dose observed over different categories of renal function.

\subsubsection{Secondary Endpoints}

Results from the analyses of secondary endpoints are presented in Table 1 . HbA1c significantly decreased early during treatment (baseline, $7.62 \pm 0.51$; Week $27.48 \pm$ $0.51 \% ; p<0.001$ ) and remained at lower levels from Week 4 throughout the 24-week treatment period. HbA1c at Week 24 was $7.32 \pm 0.51$, and the reduction from baseline was significant $(p<0.001)$. This effect was similar among the categories stratified by baseline insulin dose: $-0.29 \%$, $-0.34 \%$, and $-0.25 \%$ at end of treatment in the $<15,15$ to $<30$, and $\geq 30$ units/day categories, respectively (all $p<0.05)$. Similar results were seen for FPG and glycoalbumin (data not shown).

Although serum total cholesterol, leptin, adiponectin, and C-peptide showed no changes, glucagon levels were slightly decreased at Week 24. Body weight, and waist circumference decreased gradually throughout the treatment period, with significant changes observed by the end of the treatment period. Reductions were also observed in both systolic and diastolic blood pressure. In addition, DTSQ total score (sum of the scores of questions 1, 4, 5, 6, 7, and 8) improved significantly at Week 24 compared with baseline (Table 1).

One patient $(1.02 \%, 1 / 98)$, whose baseline insulin dose was 10 units/day, was able to stop use of insulin at Week 16 by addition of ipragliflozin.

\subsection{Safety}

Most patients (60.2\%) had TEAEs (Table 2), with the most common being nasopharyngitis $(17.5 \%)$, hypoglycemia (13.6\%), and pollakiuria (13.6\%). Hypoglycemia (10.7\%) and pollakiuria (13.6\%) were also the most common drugrelated TEAEs. No patients died during the study. All TEAEs were mild or moderate in severity, except for one event (cerebral infarction) assessed as severe and possibly related to the study drug.

Four patients withdrew from the study because of TEAEs; two patients withdrew because of serious TEAEs. One patient, a 62-year-old male, experienced a cerebral infarction on Day 85 that resolved just over 2 months later. For which, 
Fig. 1 Insulin dose during the study. a Time-course of change in insulin dose. ${ }^{*} p<0.001$ vs baseline (Wilcoxon's signed rank test). b Time-course of percent change in insulin dose. c Time-course of percent change in insulin dose stratified by baseline insulin dose $(<15$, 15 to $<30$, and $\geq 30$ units/day). All values are means and the error bars represent standard deviations $\mathbf{a}$

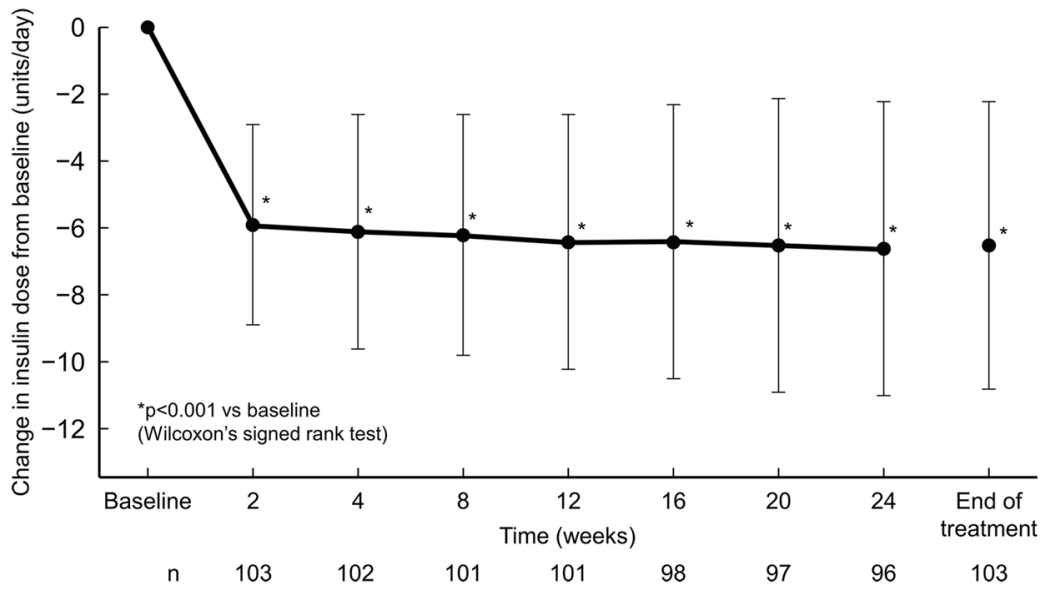

b

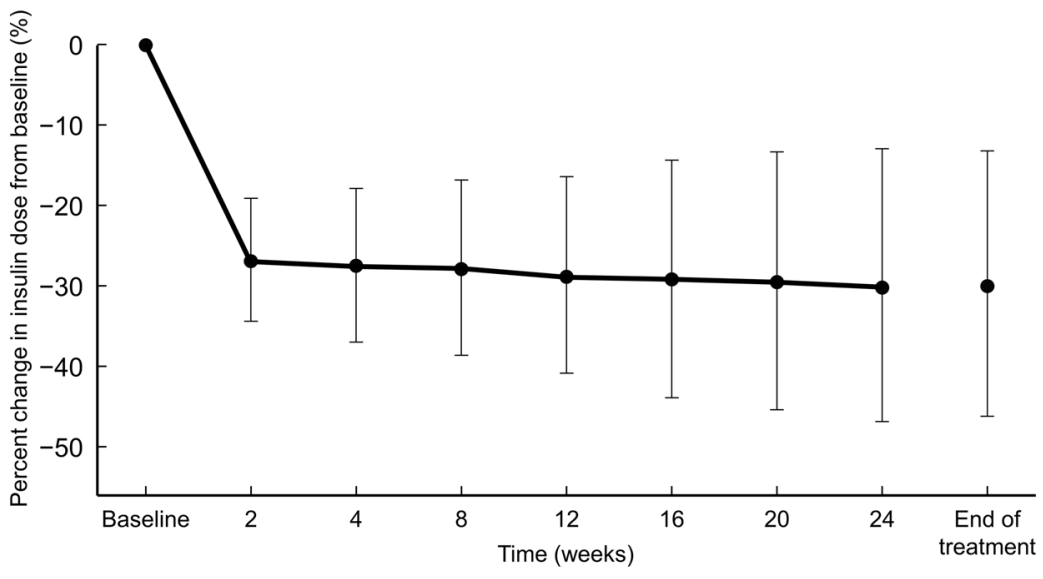

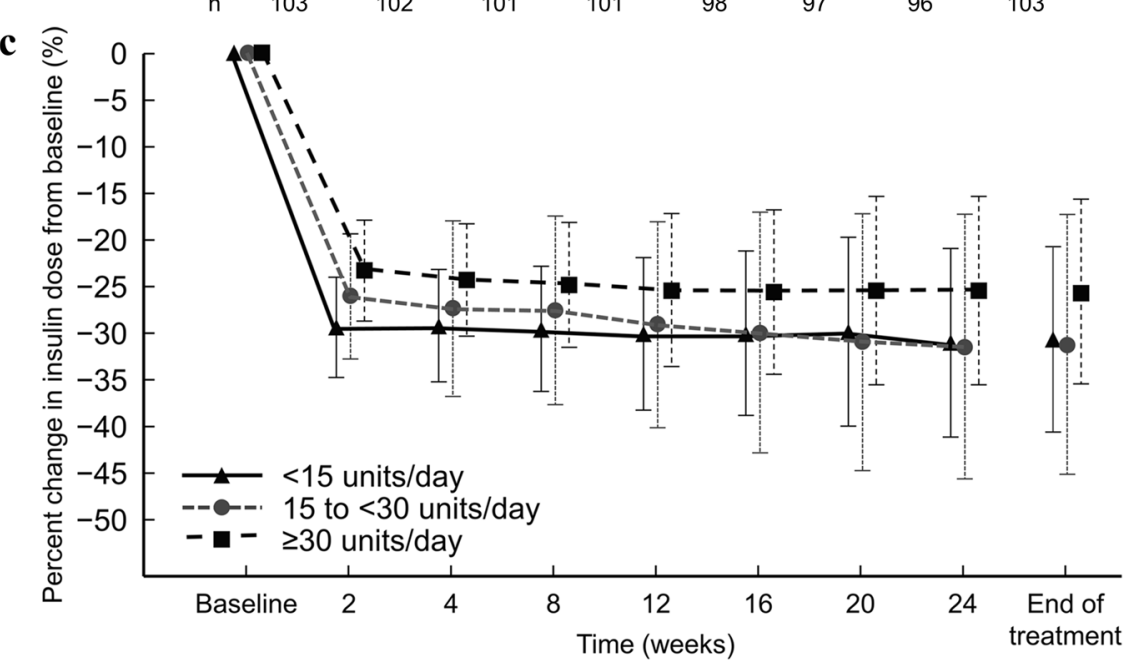
$<15$ units/day
15 to $<30$ units/day
$\geq 30$ units/day

$\begin{array}{lllllllll}37 & 37 & 36 & 36 & 36 & 35 & 35 & 34 & 37 \\ 39 & 39 & 39 & 38 & 38 & 38 & 37 & 37 & 39 \\ 27 & 27 & 27 & 27 & 27 & 25 & 25 & 25 & 27\end{array}$

hypoglycemia was discounted as the reason. This event was considered to be possibly related to ipragliflozin; therefore, the patient was withdrawn from the study. The other patient experienced a foot fracture subsequent to falling off a bicycle (no hypoglycemia) that led to insomnia and eventual study withdrawal; the patient continued ipragliflozin treatment outside the study. Two patients experienced non-serious TEAEs that led to withdrawal. These included renal impairment in a patient with a history of diabetic nephropathy and drug eruption (urticaria) in a patient that developed after Visit 2 and that remained unresolved. 
Table 1 Absolute values and changes in efficacy variables from baseline to end of treatment

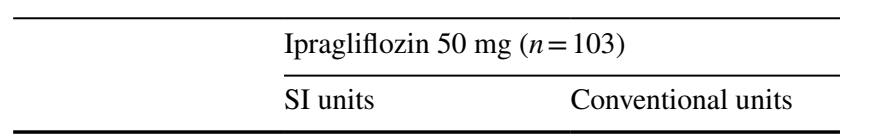

HbA1c

$\begin{array}{lll}\text { Units } & (\mathrm{mmol} / \mathrm{mol}) & (\%[\mathrm{NGSP}]) \\ \text { Baseline } & 59.7 \pm 5.6 & 7.62 \pm 0.51 \\ \text { EOT } & 56.5 \pm 5.5 & 7.32 \pm 0.51 \\ \text { Change at EOT } & -3.3 \pm 6.1, p<0.001 & -0.30 \pm 0.56 \\ & & p<0.001\end{array}$

\section{FPG}

$\begin{array}{lll}\text { Units } & (\mathrm{mmol} / \mathrm{L}) & (\mathrm{mg} / \mathrm{dL}) \\ \text { Baseline } & 7.17 \pm 1.89 & 129.2 \pm 34.1 \\ \text { EOT } & 6.70 \pm 1.46 & 120.6 \pm 26.3 \\ \text { Change at EOT } & -0.47 \pm 1.86, & -8.5 \pm 33.6, p=0.003 \\ & p=0.003 & \end{array}$

Glycoalbumin (\%)

Baseline

EOT

Change at EOT

Cholesterol

\begin{tabular}{|c|c|c|}
\hline Units & $(\mathrm{mmol} / \mathrm{L})$ & $(\mathrm{mg} / \mathrm{dL})$ \\
\hline Baseline & $4.8 \pm 0.8$ & $185.8 \pm 31.2$ \\
\hline EOT & $4.7 \pm 0.9$ & $182.6 \pm 34.0$ \\
\hline Change at EOT & $-0.1 \pm 0.8, p=0.111$ & $-3.2 \pm 24.2, p=0.111$ \\
\hline \multicolumn{3}{|l|}{ Leptin } \\
\hline Units & $(\mathrm{nmol} / \mathrm{L})$ & $(\mathrm{ng} / \mathrm{mL})$ \\
\hline Baseline & $0.90 \pm 0.51$ & $14.33 \pm 8.21$ \\
\hline EOT & $0.83 \pm 0.43$ & $13.35 \pm 6.87$ \\
\hline Change at EOT & $\begin{array}{c}-0.06 \pm 0.25 \\
p=0.089\end{array}$ & $\begin{array}{c}-0.99 \pm 3.95 \\
p=0.089\end{array}$ \\
\hline \multicolumn{3}{|l|}{ Adiponectin } \\
\hline Units & - & $(\mu \mathrm{g} / \mathrm{mL})$ \\
\hline Baseline & & $7.73 \pm 4.72$ \\
\hline EOT & & $7.74 \pm 4.43$ \\
\hline Change at EOT & & $0.00 \pm 1.57, p=0.674$ \\
\hline \multicolumn{3}{|l|}{ C-peptide } \\
\hline Units & $(\mathrm{nmol} / \mathrm{L})$ & $(\mathrm{ng} / \mathrm{mL})$ \\
\hline Baseline & $0.42 \pm 0.29$ & $1.255 \pm 0.878$ \\
\hline EOT & $0.42 \pm 0.28$ & $1.257 \pm 0.846$ \\
\hline Change at EOT & $\begin{array}{c}-0.01 \pm 0.21 \\
p=0.940\end{array}$ & $\begin{array}{c}0.003 \pm 0.625 \\
p=0.940\end{array}$ \\
\hline \multicolumn{3}{|l|}{ Glucagon } \\
\hline Units & - & $(\mathrm{pg} / \mathrm{mL})$ \\
\hline Baseline & & $44.03 \pm 21.02$ \\
\hline EOT & & $39.77 \pm 19.09$ \\
\hline Change at EOT & & $\begin{array}{c}-4.26 \pm 18.42 \\
p=0.021\end{array}$ \\
\hline
\end{tabular}

Table 1 (continued)

\begin{tabular}{|c|c|c|}
\hline & \multicolumn{2}{|c|}{ Ipragliflozin $50 \mathrm{mg}(n=103)$} \\
\hline & SI units & Conventional units \\
\hline \multicolumn{3}{|l|}{ Body weight } \\
\hline Units & $(\mathrm{kg})$ & - \\
\hline Baseline & $73.92 \pm 13.35$ & \\
\hline EOT & $71.57 \pm 13.58$ & \\
\hline Change at EOT & $\begin{array}{c}-2.35 \pm 2.10 \\
p<0.001\end{array}$ & \\
\hline \multicolumn{3}{|c|}{ Waist circumference } \\
\hline Units & $(\mathrm{cm})$ & - \\
\hline Baseline & $92.88 \pm 9.32$ & \\
\hline EOT & $91.02 \pm 9.55$ & \\
\hline Change at EOT & $\begin{array}{c}-1.86 \pm 3.48 \\
p<0.001\end{array}$ & \\
\hline \multicolumn{3}{|l|}{ SBP } \\
\hline Units & - & $(\mathrm{mmHg})$ \\
\hline Baseline & & $134.1 \pm 16.2$ \\
\hline EOT & & $128.4 \pm 15.3$ \\
\hline Change at EOT & & $-5.7 \pm 15.8, p<0.001$ \\
\hline \multicolumn{3}{|l|}{ DBP } \\
\hline Units & - & $(\mathrm{mmHg})$ \\
\hline Baseline & & $77.8 \pm 9.9$ \\
\hline EOT & & $75.5 \pm 10.6$ \\
\hline Change at EOT & & $-2.3 \pm 9.1, p=0.002$ \\
\hline \multicolumn{3}{|l|}{ DTSQ total score } \\
\hline Baseline & - & $25.3 \pm 6.3$ \\
\hline EOT & & $27.4 \pm 6.1$ \\
\hline Change at EOT & & $2.1 \pm 6.2, p<0.001$ \\
\hline
\end{tabular}

Values are presented as the mean \pm standard deviation

$D B P$ diastolic blood pressure, DTSQ Diabetes Treatment Satisfaction Questionnaire, EOT end of the treatment period, $F P G$ fasting plasma glucose, HbAlc glycated hemoglobin, NGSP National Glycohemoglobin Standardization Program, SBP systolic blood pressure, SI units International System of Units

${ }^{\mathrm{a}} n=101$

There were no notable or clinically significant changes in hematology or biochemistry laboratory test results during the study, with the exception of acetoacetic acid, 3-hydroxybutyric acid, and ketone bodies. The mean values for these increased notably (175-200\% of baseline) at Week 2 but were stable thereafter.

\section{Discussion}

In the present study, the insulin dose was successfully reduced by approximately $30 \%$ of the baseline dose, and glycemic control was maintained in this cohort of patients with 
Table 2 Treatment-emergent adverse events (TEAEs) by system organ class and preferred term ${ }^{\mathrm{a}}$ (safety analysis set)

\begin{tabular}{|c|c|c|}
\hline & \multicolumn{2}{|c|}{$\begin{array}{l}\text { Ipragliflozin } 50 \mathrm{mg} \\
(n=103)\end{array}$} \\
\hline & $n(\%)$ & Events \\
\hline \multicolumn{3}{|l|}{ Overview of TEAEs } \\
\hline TEAEs & $62(60.2)$ & 174 \\
\hline Drug-related $^{\mathrm{b}}$ TEAEs & $34(33.0)$ & 95 \\
\hline Serious TEAEs & $2(1.9)$ & 2 \\
\hline Drug-related ${ }^{\mathrm{b}}$ serious TEAEs & $1(1.0)$ & 1 \\
\hline $\begin{array}{l}\text { TEAEs leading to permanent dis- } \\
\text { continuation }\end{array}$ & $4(3.9)$ & 4 \\
\hline $\begin{array}{l}\text { Drug-related }{ }^{b} \text { TEAEs leading to } \\
\text { permanent discontinuation }\end{array}$ & $2(1.9)$ & 2 \\
\hline Deaths & 0 & 0 \\
\hline \multicolumn{3}{|c|}{ Common TEAEs occurring in $\geq 2 \%$ of patients } \\
\hline \multicolumn{3}{|c|}{ System organ class } \\
\hline \multicolumn{3}{|l|}{ Preferred term } \\
\hline Overall & $40(38.8)$ & 112 \\
\hline \multicolumn{3}{|l|}{ Gastrointestinal disorders } \\
\hline Constipation & $6(5.8)$ & 6 \\
\hline \multicolumn{3}{|c|}{ General disorders and administration site conditions } \\
\hline Thirst & $5(4.9)$ & 5 \\
\hline \multicolumn{3}{|l|}{ Infections and infestations } \\
\hline Nasopharyngitis & $18(17.5)$ & 28 \\
\hline \multicolumn{3}{|l|}{ Metabolism and nutrition disorders } \\
\hline Hypoglycemia & $14(13.6)$ & 58 \\
\hline \multicolumn{3}{|l|}{ Renal and urinary disorders } \\
\hline Pollakiuria & $14(13.6)$ & 14 \\
\hline \multicolumn{3}{|l|}{ Drug-related TEAEs } \\
\hline \multicolumn{3}{|l|}{ System organ class } \\
\hline \multicolumn{3}{|l|}{ Preferred term } \\
\hline Overall & $34(33.0)$ & 95 \\
\hline Gastrointestinal disorders & $8(7.8)$ & 9 \\
\hline Constipation & $6(5.8)$ & 6 \\
\hline Vomiting & $2(1.9)$ & 2 \\
\hline Periodontal disease & $1(1.0)$ & 1 \\
\hline $\begin{array}{l}\text { General disorders and administration } \\
\text { site conditions }\end{array}$ & $5(4.9)$ & 5 \\
\hline Thirst & $5(4.9)$ & 5 \\
\hline Investigations & $2(1.9)$ & 2 \\
\hline Weight decreased & $1(1.0)$ & 1 \\
\hline Blood ketone body increased & $1(1.0)$ & 1 \\
\hline Metabolism and nutrition disorders & $13(12.6)$ & 56 \\
\hline Hypoglycemia & $11(10.7)$ & 54 \\
\hline Ketosis & $1(1.0)$ & 1 \\
\hline Dyslipidemia & $1(1.0)$ & 1 \\
\hline $\begin{array}{l}\text { Musculoskeletal and connective tis- } \\
\text { sue disorders }\end{array}$ & $1(1.0)$ & 1 \\
\hline Musculoskeletal discomfort & $1(1.0)$ & 1 \\
\hline Nervous system disorders & $3(2.9)$ & 3 \\
\hline Cerebral infarction & $1(1.0)$ & 1 \\
\hline
\end{tabular}

Table 2 (continued)

\begin{tabular}{llc}
\hline & \multicolumn{1}{l}{$\begin{array}{l}\text { Ipragliflozin 50 mg } \\
(n=103)\end{array}$} & Events \\
\cline { 2 - 3 } & $n(\%)$ & 1 \\
\hline Dizziness & $1(1.0)$ & 1 \\
Hypoesthesia & $1(1.0)$ & 14 \\
Renal and urinary disorders & $14(13.6)$ & 14 \\
$\quad$ Pollakiuria & $14(13.6)$ & 2 \\
Reproductive system and breast & $2(1.9)$ & 2 \\
disorders & $2(1.9)$ & 3 \\
$\quad$ Pruritus genital & $3(2.9)$ & 1 \\
Skin and subcutaneous tissue & & 1 \\
disorders & $1(1.0)$ & 1 \\
$\quad$ Dermatitis contact & $1(1.0)$ & \\
$\quad$ Drug eruption & $1(1.0)$ & \\
$\quad$ Rash &
\end{tabular}

Values are shown as $n(\%)$ (with $n$ indicating number of subjects) and number of events

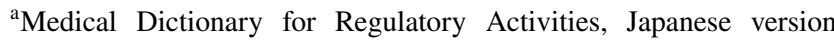
(MedDRA/J v19.0)

${ }^{\mathrm{b}}$ Possible, probable or unknown, as assessed by the investigator, or records where relationship was unknown

moderate control at baseline. The percent insulin dose reductions during the study were similar across all three baseline insulin dose categories. The current study also demonstrated that the mean DTSQ total score improved by over 2 points, suggesting that patients' treatment satisfaction may improve when insulin doses are reduced. This is an important consideration because of the long-term nature of insulin therapy for the majority of patients with T2DM.

It was intriguing to find that percent reductions in insulin dose and HbA1c improvement were similar across all three baseline insulin dose categories. In addition, a reduction in insulin dose in patients with BMIs $<25 \mathrm{~kg} / \mathrm{m}^{2}$ was similar to those with BMIs $\geq 25 \mathrm{~kg} / \mathrm{m}^{2}$ (-30.45\% and $-29.58 \%$, respectively). Furthermore, the insulin-sparing effects of ipragliflozin was similar in patients aged $<65$ years and those aged $\geq 65$ years $(-30.30 \%$ and $-28.88 \%$, respectively). Diabetic states, including residual pancreatic $\beta$-cell mass, may differ between these patients. It can be hypothesized that ipragliflozin can exert an insulin-sparing effect regardless of a patients' diabetic state, including residual $\beta$-cell function. While this is noteworthy, care is warranted as some patients with reduced $\beta$-cell function may be at a higher risk of euglycemic diabetic ketoacidosis [21] caused by insulin deficiency and dehydration [22]. Although this study examined the insulin-sparing effects of ipragliflozin in T2DM, it is advised that extra caution be used in patients with type 1 diabetes mellitus [23, 24].

Retrospective studies have shown that SGLT2 inhibitors reduced insulin doses by $10 \%$ and $20 \%$ in real-world treatment studies conducted in Canada [15] and India [16], 
respectively. However, these reports were based on insurance databases and retrospective clinical chart review rather than prospective controlled clinical trial data. Many differing factors between these studies could have led to the different results, including use of combined therapies, obesity status and patient race/ethnicity. The use of dietary interventions, which can strongly influence the effectiveness of SGLT2 inhibitors, is likely to be different between prospective and retrospective study types. Prospective studies with designs that are similar to the present study are needed to clarify factors that affect the insulin-sparing effect of SGLT2 inhibitors.

The incidence of hypoglycemia in the current study was low (10.7\%), with no severe cases of hypoglycemia reported. Although it is critical to remember the limitations in making any direct comparisons between studies, it is worth mentioning that the incidence of hypoglycemia in the current study was approximately one-third of a previous study (IOLITE) in which patients received ipragliflozin add-on therapy to insulin [25]. During the double-blind period of the IOLITE study, $29.1 \%$ of ipragliflozin-treated patients experienced hypoglycemia versus $14.9 \%$ of patients who received placebo [25]. In the IOLITE study, ipragliflozin therapy was initiated without a prior reduction of insulin dose and an $\mathrm{HbA} 1 \mathrm{c}$ reduction of $1.0 \%$ was achieved. It should also be noted that inclusion criteria for baseline $\mathrm{HbAlc}$ levels differed in these studies: $6.5 \%$ to $<8.0 \%$ in the present study versus $7.5 \%$ to $\leq 10.5 \%$ in the IOLITE study. Nonetheless, our data suggest that a $30 \%$ dose reduction of insulin is recommended for safety and efficacy reasons when $50-\mathrm{mg}$ ipragliflozin is added on to insulin therapy.

Patients exhibited significant decreases in body weight and waist circumference at the end of the study. Significant reductions were also observed in both systolic and diastolic blood pressure. These data illustrate the beneficial effect of ipragliflozin on cardiovascular risk factors, a result that is in line with previous reports of other SGLT2 inhibitors [7, 8].

The limitations of the current study include those inherent to single-arm, open-label studies. Our study enrolled only Japanese patients, so the generalizability to other ethnic populations is limited. Additionally, the strict protocol-specified dose adjustment criteria for insulin may have proved challenging for some physicians. More flexible dosing criteria could have resulted in additional or greater insulin dose reductions. Finally, our study was limited in that the sample size was relatively small.

\section{Conclusions}

In conclusion, in patients with T2DM treated with basal insulin, the addition of once-daily 50-mg ipragliflozin for maintaining $\mathrm{HbA} 1 \mathrm{c}$ levels enabled a statistically significant dose reduction (approximately 30\%) of insulin by Week 24 compared with baseline. The percent reductions in insulin dose during the study were similar across all three baseline insulin dose categories. No major safety concerns were associated with the administration of once-daily 50-mg ipragliflozin for 24 weeks in combination with insulin therapy. Further research should investigate whether an ipragliflozin dose increase to $100 \mathrm{mg}$ daily could further reduce insulin dose or facilitate withdrawal from insulin entirely in patients with T2DM.

Acknowledgments The authors thank Michelle Belanger, MD, and Mary Richardson, MSc, of Edanz Medical Writing for providing medical writing support, which was funded by Astellas through EMC K.K. in accordance with Good Publication Practice (GPP3) guidelines (http://www.ismpp.org/gpp3). The authors also acknowledge the contributions of Ikko Nakao of Astellas Pharma Inc., Japan, who assisted with the study design, study conduct, data collection and analysis, and writing of the manuscript.

\section{Compliance with Ethical Standards}

Ethical approval This study was conducted in accordance with the ethical principles of the Declaration of Helsinki, Good Clinical Practice, Good Post-marketing Surveillance Practice, and the International Council for Harmonization of Technical Requirements for Pharmaceuticals for Human Use Guidelines. Institutional review boards at each site approved the study.

Informed consent All patients provided informed consent at Visit 1 before any study-related screening procedures commenced.

Funding This study was funded by Astellas Pharma Inc.

Conflict of interest $\mathrm{HI}$ has served on the scientific advisory board of Astellas Pharma Inc.; received lecture or consulting fees from Astellas Pharma Inc., MSD, Sanofi, Mitsubishi Tanabe Pharma, Boehringer Ingelheim Japan, and Novartis Pharma; and received grants/research support from Astellas Pharma Inc., Ono Pharmaceutical, Boehringer Ingelheim Japan, AstraZeneca, Sanofi, Mitsubishi Tanabe Pharma, Eli Lilly Japan, Daiichi-Sankyo, Novo Nordisk Pharma, Kyowa Hakko Kirin, and MSD. SY, TS, and YK are employees of the study sponsor.

Open Access This article is distributed under the terms of the Creative Commons Attribution-NonCommercial 4.0 International License (http://creativecommons.org/licenses/by-nc/4.0/), which permits any noncommercial use, distribution, and reproduction in any medium, provided you give appropriate credit to the original author(s) and the source, provide a link to the Creative Commons license, and indicate if changes were made.

\section{References}

1. Marín-Peñalver JJ, Martín-Timón I, Sevillano-Collantes C, Del Cañizo-Gómez FJ. Update on the treatment of type 2 diabetes mellitus. World J Diabetes. 2016;7(17):354-95.

2. Hayashino $Y$, Izumi $K$, Okamura $S$, Nishimura R, Origasa H, Tajima N, et al. Duration of diabetes and types of diabetes therapy in Japanese patients with type 2 diabetes: the Japan Diabetes 
Complication and its Prevention prospective study 3 (JDCP study 3). J Diabetes Investig. 2017;8(2):243-9.

3. Brown A, Guess N, Dornhorst A, Taheri S, Frost G. Insulin-associated weight gain in obese type 2 diabetes mellitus patients: what can be done? Diabetes Obes Metab. 2017;19(12):1655-68.

4. Erpeldinger S, Rehman MB, Berkhout C, Pigache C, Zerbib Y, Regnault $\mathrm{F}$, et al. Efficacy and safety of insulin in type 2 diabetes: meta-analysis of randomised controlled trials. BMC Endocr Disord. 2016;16(1):39.

5. Home P, Riddle M, Cefalu WT, Bailey CJ, Bretzel RG, Del Prato $\mathrm{S}$, et al. Insulin therapy in people with type 2 diabetes: opportunities and challenges? Diabetes Care. 2014;37(6):1499-508.

6. Lewey J, Wei W, Lauffenburger JC, Makanji S, Chant A, DiGeronimo J, et al. Targeted Adherence Intervention to Reach Glycemic Control with Insulin Therapy for patients with Diabetes (TARGIT-Diabetes): rationale and design of a pragmatic randomised clinical trial. BMJ Open. 2017;7(10):e016551.

7. Zinman B, Wanner C, Lachin JM, Fitchett D, Bluhmki E, Hantel $\mathrm{S}$, et al. Empagliflozin, cardiovascular outcomes, and mortality in type 2 diabetes. N Engl J Med. 2015;373(22):2117-28.

8. Neal B, Perkovic V, Mahaffey KW, de Zeeuw D, Fulcher G, Erondu N, et al. Canagliflozin and cardiovascular and renal events in type 2 diabetes. N Engl J Med. 2017;377(7):644-57.

9. Ishihara H, Yamaguchi S, Nakao I, Asahina S, Sakatani T. Efficacy and safety of ipragliflozin as add-on therapy to insulin in Japanese patients with type 2 diabetes mellitus (IOLITE): a 36-week, openlabel extension of a 16-week, randomized, placebo-controlled, double-blind study. Diabetol Int. 2019;10(1):37-50.

10. Ishihara H, Yamaguchi S, Nakao I, Sakatani T. Ipragliflozin addon therapy to a glp-1 receptor agonist in japanese patients with type 2 diabetes (AGATE): a 52-week open-label study. Diabetes Ther. 2018;9(4):1549-67.

11. Nakamura I, Maegawa H, Tobe K, Tabuchi H, Uno S. Safety and efficacy of ipragliflozin in Japanese patients with type 2 diabetes in real-world clinical practice: interim results of the STELLALONG TERM post-marketing surveillance study. Expert Opin Pharmacother. 2018;19(3):189-201.

12. Kashiwagi A, Sakatani T, Nakamura I, Akiyama N, Kazuta K, Ueyama E, et al. Improved cardiometabolic risk factors in Japanese patients with type 2 diabetes treated with ipragliflozin: a pooled analysis of six randomized, placebo-controlled trials. Endocr J. 2018;65(7):693-705.

13. Wilding JP, Woo V, Soler NG, Pahor A, Sugg J, Rohwedder K, et al. Long-term efficacy of dapagliflozin in patients with type 2 diabetes mellitus receiving high doses of insulin: a randomized trial. Ann Intern Med. 2012;156(6):405-15.

14. Neal B, Perkovic V, de Zeeuw D, Mahaffey KW, Fulcher G, Ways K, et al. Efficacy and safety of canagliflozin, an inhibitor of sodium-glucose cotransporter 2 , when used in conjunction with insulin therapy in patients with type 2 diabetes. Diabetes Care. 2015;38(3):403-11.

15. Harris SB, Mequanint S, Miller K, Reichert SM, Spaic T. When insulin therapy fails: the impact of SGLT2 inhibitors in patients with type 2 diabetes. Diabetes Care. 2017;40(10):e141-2.

16. Sosale B, Sosale A, Bhattacharyya A. Clinical effectiveness and impact on insulin therapy cost after addition of dapagliflozin to patients with uncontrolled type 2 diabetes. Diabetes Ther. 2016;7(4):765-76.

17. Bradley C. The DTSQ rev 11.8.15, DTSQ Summary HPR1882. https://www.healthpsychologyresearch.com/sites/default/files /guidelines/DTSQ\%20Summary\%20rev_11.8.15_web.pdf. Accessed 24 May 2019.

18. Rosenstock J, Jelaska A, Zeller C, Kim G, Broedl UC, Woerle HJ, et al. Impact of empagliflozin added on to basal insulin in type 2 diabetes inadequately controlled on basal insulin: a 78-week randomized, double-blind, placebo-controlled trial. Diabetes Obes Metab. 2015;17(10):936-48.

19. Rosenstock J, Jelaska A, Frappin G, Salsali A, Kim G, Woerle HJ, et al. Improved glucose control with weight loss, lower insulin doses, and no increased hypoglycemia with empagliflozin added to titrated multiple daily injections of insulin in obese inadequately controlled type 2 diabetes. Diabetes Care. 2014;37(7):1815-23.

20. Wilding JPH, Woo V, Rohwedder K, Sugg J, Parikh S, Dapagliflozin 006 Study Group. Dapagliflozin in patients with type 2 diabetes receiving high doses of insulin: efficacy and safety over 2 years. Diabetes Obes Metab. 2014;16(2):124-36.

21. Ogawa W, Sakaguchi K. Euglycemic diabetic ketoacidosis induced by SGLT2 inhibitors: possible mechanism and contributing factors. J Diabetes Investig. 2016;7(2):135-8.

22. Perry RJ, Rabin-Court A, Song JD, Cardone RL, Wang Y, Kibbey RG, et al. Dehydration and insulinopenia are necessary and sufficient for euglycemic ketoacidosis in SGLT2 inhibitor-treated rats. Nat Commun. 2019;10(1):548

23. Ogawa W, Hirota Y. Sodium-glucose cotransporter 2 inhibitorassociated diabetic ketoacidosis in patients with type 1 diabetes: metabolic imbalance as an underlying mechanism. J Diabetes Investig. 2019;10(4):879-82.

24. Kaku K, Isaka H, Sakatani T, Toyoshima J. Efficacy and safety of ipragliflozin add-on therapy to insulin in Japanese patients with type 1 diabetes mellitus: a randomized, double-blind, phase 3 trial. Diabetes Obes Metab. 2019. (Epub ahead of print) (in press).

25. Ishihara H, Yamaguchi S, Nakao I, Okitsu A, Asahina S. Efficacy and safety of ipragliflozin as addon therapy to insulin in Japanese patients with type 2 diabetes mellitus (IOLITE): a multicentre, randomized, placebo-controlled, double-blind study. Diabetes Obes Metab. 2016;18(12):1207-16. 